\title{
FLEXOR HALLUX TENDON TRANSFER: COMPARATIVE STUDY THROUGH DOUBLE OR SINGLE APPROACH
}

\author{
Marcos Hideyo Sakaki ${ }^{1}$, Alexandre Leme Godoy-Santos ${ }^{1}$, Rafael Trevisan Ortiz ${ }^{1}$, Antônio Araújo ${ }^{2}$, Túllo Diniz Fernandes ${ }^{1}$
}

\section{ABSTRACT}

Objective: To quantify the FHL length difference obtained through a single approach and by a double combined approach. Methods: 16 fresh cadavers, a total of 32 feet, were used to measure the FHL graft length. With the cadaver positioned in ventral decubitus, a posteromedial incision in the ankle and a second incision in the plantar cavus were performed. Results: The average gain of tendon's length (GTL) was of $42.43 \mathrm{~mm}$, the lowest value being $32 \mathrm{~mm}$ and the largest $48 \mathrm{~mm}$. The comparative analysis of the GTL on the right and left sides through the paired " $\mathrm{t}$ " Test does not show statistical differences, with a p-value $=0.463$ and a statistical power of 0.1443 . The height analysis of the sample and the right and left GTL performed through linear regression do not show statistically significant differences, with a p-value of 0.38311 and 0.82640 , respectively. Conclusion: Harvesting the FHL graft using a double combined approach yields a $42.43 \mathrm{~mm}$ length gain in comparison to harvesting using the single approach. Level of Evidence III, Case Control Study.

Keywords: Tendinopathy. Hallux. Achilles tendon.

Citation: Sakaki MH, Godoy-Santos AL, Ortiz RT, Araújo A, Fernandes TD. Flexor hallux tendon transfer: comparative study through double or single approach. Acta Ortop Bras. [online]. 2014;22(3):140-3. Available from URL: http://www.scielo.br/aob.

\section{INTRODUÇÃO}

Nonsurgical treatment of chronic tendinopathy of the Achilles tendon (TAT) is the initial form of approach and includes indications such as rest, anti-inflammatory medications, correction of extrinsic factors, stretching the sural triceps, and use of orthoses to alleviate the influence of intrinsic factors, among others. When conservative treatment is not well succeed, surgery is indicated, and injured Achilles tendon may be retained or replaced depending on its degree of degeneration. ${ }^{1}$ In cases of resection of the Achilles tendon, using the flexor hallux longus tendon (FHL) as substitute has been used frequently in medical literature for presenting some advantages over other tendons available. They are: the long flexor hallux muscle goes into action at the same stage as the sural triceps during gait; ${ }^{2}$ it has twice and $30 \%$ more power than the flexor digitorum longus and fibularis brevis flexor, respectively; ${ }^{3}$ its axis contraction is the same as the sural triceps; ${ }^{4}$ it can be dissected with the same approach as that used in the treatment of tendinopathy of the Achilles tendon; ${ }^{5}$ and its muscular body sutured to the Achill tendon serves as a vascularization source for its regeneration. ${ }^{6}$ Two dissection techniques for obtaining $\mathrm{FHL}$ are described, one in the posterior segment of the distal leg, the same used for the treatment of chronic tendinopathy of the Achilles ten- don, through which the tendon is cut along the osteofibrous tunnel located after the subsequent process the talus, after being pulled upwards with the foot and hallux in maximum plantar flexion to allow obtaining the greatest possible tendon length. ${ }^{6}$ The other technique involves the preparation of a second access track to medial plantar cavus, in addition to the first way approach to the Achilles tendon, whereby the $\mathrm{FHL}$ is identified and transected immediately proximal to Henry's knot, and then pulled through the proximal track. ${ }^{2}$ This procedure allows to obtain a longer tendon graft at the expense of a second incision with longer exposure and higher risk of injury to the neurovascular structures located near the plantar tendon. TAT is treated with graft $\mathrm{FHL}$ obtained by both single track and double track combined, with similar clinical outcomes. ${ }^{1,7-14}$ In an anatomical study on cadavers, Tashjian et $\mathrm{al} .{ }^{15}$ found that the average length of $\mathrm{FHL}$ obtained by the unique track is $5.16 \mathrm{~cm}$, while on the dual track is 8.09 $\mathrm{cm}$. It is not clear whether the increase in length of $\mathrm{FHL}$ achieved by combined dual track brings any benefits to the reconstruction of TAT.

The objective of this study is to quantify the difference in length of $\mathrm{FHL}$ obtained by single track and double track combined in the Brazilian population.

All the authors declare that there is no potential conflict of interest referring to this article.

\footnotetext{
1. Institute of Orthopedics and e Traumatology, Hospital das Clínicas, Faculdade de Medicina, Universidade de São Paulo, São Paulo, SP, Brazil. 2. Hospital São Lucas, Natal, RN, Brazil.

Work developed at LIM 41 - Laboratory of Medical Research of the Musculoskeletal System and Osteometabolic and Degenerative diseases, Department of Orthopedics and Traumatology, Faculdade de Medicina da Universidade de São Paulo, São Paulo, SP, Brazil.

Correspondence: Rua Dr. Ovídio Pires de Campos, 333, Cerqueira Cesar, 05403-010 São Paulo, SP, Brazil. alexandrelemegodoy@gmail.com.br
} 


\section{MATERIALS AND METHODS}

After approval by the Scientific Committee and the Ethics Committee of the University 16 fresh cadavers, totalizing 32 feet, were used to measure the length of the FHL graft. All cadavers were male, aged 25-45 years with no history or signs of previous injuries in the foot and ankle region. The height of the cadavers ranged $167-182 \mathrm{~cm}$, with a mean of $173 \mathrm{~cm}$ and body weight ranged $62-78 \mathrm{~kg}, 70 \mathrm{~kg}$ on average.

With the corpse placed in the prone position, one posteromedial longitudinal incision was made in the ankle and, after opening the flexor retinaculum, FHL was identified and isolated from the muscle-tendon transition to the entrance of the osteofibrous tunnel posterior to the talus. (Figure 1A)

FHL was pulled upwards while the foot and hallux were placed in maximum plantar flexion, a maneuver which is typically performed in the surgical field for obtaining the greatest possible length of $\mathrm{FHL}$ when using only one access track. In this condition, a mark was made on the tendon with a surgical pen on its lower visible limit, adjacent to osteofibrous tunnel, corresponding to the point where the tenotomy is held in single track surgeries.

A second longitudinal incision was made in plantar cavus in the navicular topography and the middle compartment of the foot was accessed to identify Henry's node. FHL was isolated and transected immediately proximal to the node and retracted by the first access track. (Figure 1B)

With FHL stretched and supported on a flat surface, three measurements were made with a millimeter ruler of the distance between the most proximal portion of the pen mark to the most proximal portion of the edge of the sectioned tendon, and the average of the measurements was taken. (Figure 1C)

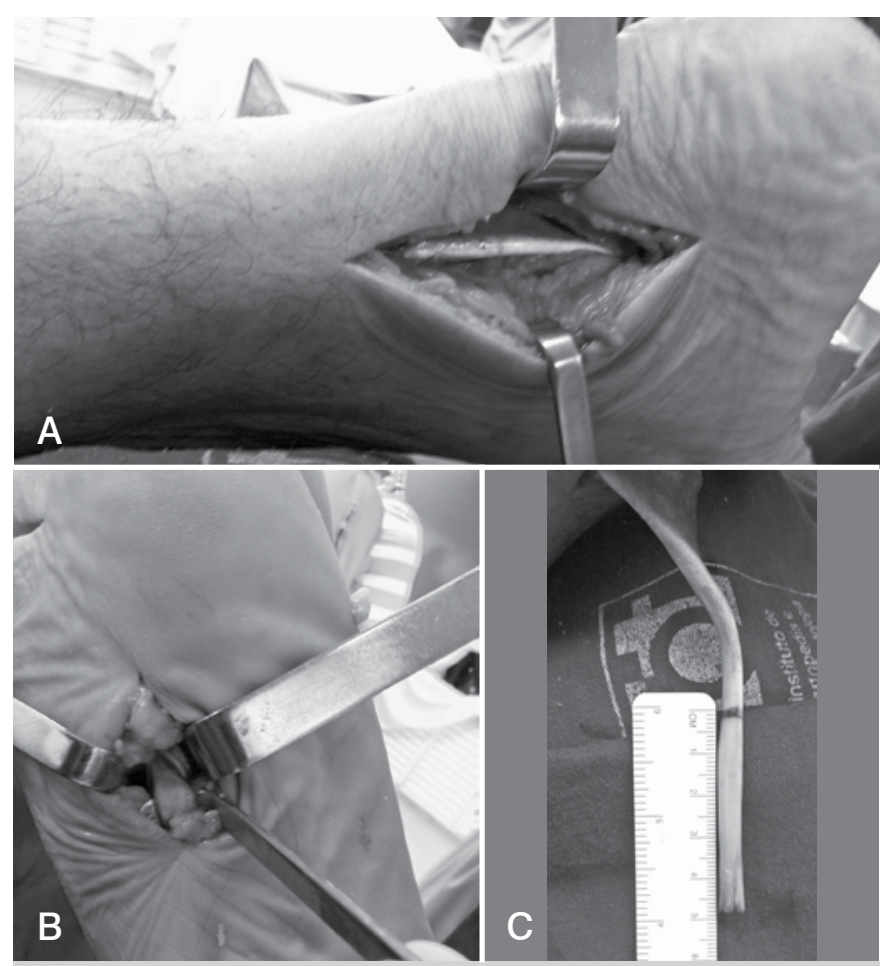

Figure 1. (A) FHL visualization through the posteromedial access track (B) FHL visualization via plantarmedial access (C) Measurement using a millimeter ruler of the difference of the flexor hallux tendon transfer graft accessed by posteromedial and medial plantar access tracks
It represents the length difference of FHL to be transferred through single and dual access track, which we call gain of FHL tendon's graft length (GTL).

\section{RESULTS}

Table 1 shows the GLG obtained from 16 cadavers, totalizing 32 measurements, being 16 right and 16 left. Table 2 shows the descriptive analysis of findings of the study.

The average gain in length of the graft tendon flexor hallux was $42.43 \mathrm{~mm}$, the lowest being $32 \mathrm{~mm}$ and the higher $48 \mathrm{~mm}$.

A comparative evaluation between the right and left sides using paired " $\mathrm{t}$ " Test shows no statistical difference between them, revealing $p=0.463$.

The correlation between the sample height and the left and right GLG performed by linear regression shows no statistical difference, $p=0.38311$ and $p=0.82640$, respectively.

Table 1. Distribution of height and FHL gain of tendon's length (GTL) on the sample studied.

\begin{tabular}{c|c|c|c}
\hline Cadaver & Height $(\mathbf{c m})$ & GTL right side $(\mathbf{m m})$ & GTL left side $(\mathbf{m m})$ \\
\hline 1 & 180 & 35 & 38 \\
\hline 2 & 178 & 32 & 32 \\
\hline 3 & 170 & 46 & 44 \\
\hline 4 & 172 & 47 & 42 \\
\hline 5 & 168 & 45 & 47 \\
\hline 6 & 179 & 47 & 47 \\
\hline 7 & 174 & 39 & 37 \\
\hline 8 & 167 & 46 & 45 \\
\hline 9 & 182 & 48 & 48 \\
\hline 10 & 170 & 38 & 37 \\
\hline 11 & 178 & 37 & 37 \\
\hline 12 & 168 & 43 & 41 \\
\hline 13 & 169 & 42 & 42 \\
\hline 14 & 173 & 45 & 45
\end{tabular}

Table 2. Descriptive distribution of analyzed parameters.

\begin{tabular}{c|c|c|c}
\hline & Height (cm) & GT right (mm) & GT left (mm) \\
\hline Mean & 173.5000 & 42.4375 & 42.0625 \\
\hline Standard deviation & 5.0728 & 4.8023 & 4.7395 \\
\hline Standard error & 1.2682 & 1.2005 & 1.1849 \\
\hline Median & 172.5 & 44.5 & 42.5 \\
\hline Minimum & 167.0 & 32.0 & 32.0 \\
\hline Maximum & 182.0 & 48.0 & 48.0 \\
\hline Total & 16 & 16 & 16 \\
\hline
\end{tabular}

\section{DISCUSSION}

The $42 \mathrm{~mm}$ GLG found when using the second access track to $\mathrm{FHL}$ section in the plantar region is greater than the $29.3 \mathrm{~mm}$ length found by Tashijan et al. ${ }^{15}$ However, their methodology is totally different, since FHL was first sectioned in the transition to the post-talus osteofibrous tunnel and they considered the measure of the available tendon as a graft. In order to calculate this measure, the authors used as reference points the top edge of the tuberosity of the calcaneus, with the ankle in neutral position, and the end of the proximal stump of the transected tendon, 
keeping a light pull on the FHL. The average value found in 14 cadavers was $51.6 \mathrm{~mm}$. In order to calculate the length of tendon graft available when using double track, to this value it was added the length of the tendon measured from the point of section to Henry's node, reaching $80.9 \mathrm{~mm}$. We believe that the length difference found between our study and the study of Tashjian et al. ${ }^{15}$ is due to the elimination, in the current work, of subjectivity on the traction force applied to FHL during measurement of tendon available for graft, since the applied strength was not measured, and also to the different population studied.

One issue that should be discussed is which is the advantage of having available $42 \mathrm{~mm}$ extra tendon in the reconstruction of Achilles tendon. Making a second track to isolation of $\mathrm{FHL}$ in the plantar region of the foot leads to increased surgical time, besides being a laborious dissection. FHL is located on the medial plantar compartment of the foot near the medial plantar neurovascular bundle, and to be accessed it is required to open the medial intermuscular septum. The retraction of the tendon to the proximal incision endangers both the medial plantar nerve as planting lateral. ${ }^{5}$

Some authors consider as an advantage of the double track technique the possibility of performing a tenodesis between the distal stump of the FHL and the flexor digitorum longus tendon to decrease the weakening of hallux flexion strength. ${ }^{16}$ In a study using pedobarograph by Coull et al..$^{17}$ found no differences in the feet where the FHL was isolated by single or double track. This conclusion, however, was not significant due to the small number of cases operated. All TAT patients treated using FHL, either by single track ${ }^{1,4,9,18}$ or double track with FHL tenodesis of flexor digitorum longus ${ }^{11-14,16}$ had loss of flexion strength of the hallux when examined, however this loss is rarely a functional deficit. The clinical results were similar with both techniques, but cannot be compared, because prospective studies have not been found comparing the complications of using single track with double track.

On surgical treatment of insertional Achilles tendinopathy, transferred FHL should be reinserted into the calcaneus bone, as there is no remaining distal stump of the Achilles tendon to be used. If reinsertion is done by making a transverse bone tunnel along the axis of the calcaneal tuberosity at $10 \mathrm{~mm}$ from the upper limit, ${ }^{14}$ and considering that the average width of the calcaneus in this region is $27 \mathrm{~mm},{ }^{19}$ to which the tendon is sutured in itself, a length of at least $74 \mathrm{~mm}$ would be needed. (Figure 2) Considering that the length of available graft taken by single track is $51 \mathrm{~mm},{ }^{15}$ this reintegration technique can only be accomplished with the removal of the graft by $\mathrm{FHL}$ by double track, as in this condition we have a $42 \mathrm{~mm}$ GTL. Using an interference screw in the calcaneus FHL insertion, ${ }^{3}$ the available tendon length required is much smaller. Using $23 \mathrm{~mm}$ screws is suitable, ${ }^{9}$ it only requires a bone tunnel filled with 30 $\mathrm{mm}$ tendon (Figure 3) a length seamlessly obtained by single track. Counting on a $42 \mathrm{~mm}$ GTL of in double track, $30 \mathrm{~mm}$ gain becomes embedded in the tunnel in retrograde direction (Figure 4) with only $12 \mathrm{~mm}$ available by the upper limit of the calcaneal tuberosity. We consider that this short tendon segment is usually not useful in rebuilding the insertional TAT, not offsetting the risk of carrying out a second access track on the foot. In non-insertional Achilles tendinopathy, the FHL graft is sutured primarily in the distal stump of the tendon and then in the remaining proximal stump of the Achilles tendon,

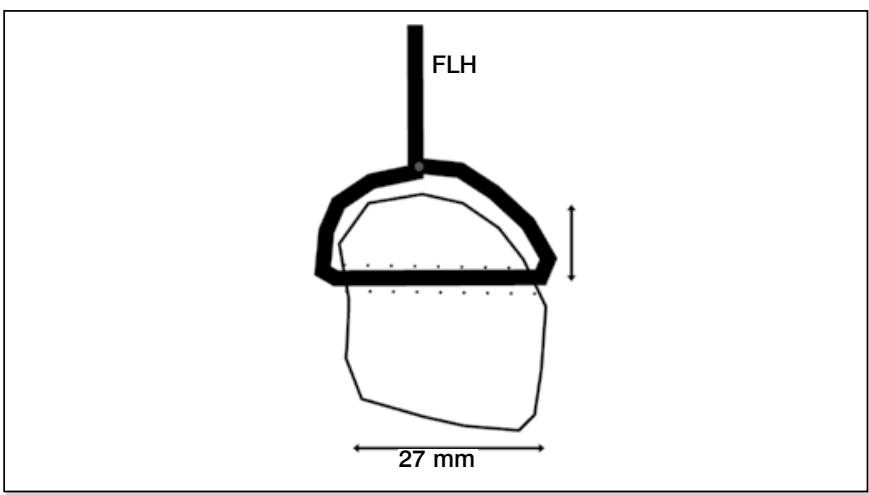

Figure 2. Posterior view of the calcaneus representing $\mathrm{FHL}$ reintegration through a bone tunnel (dotted line) made across the bone. Considering a $27 \mathrm{~mm}$ width and a $10 \mathrm{~mm}$ distance from the tunnel to the upper limit of the bone, the tendon length required for a handle (beginning and ending on the red spot) is at least $74 \mathrm{~mm}$.

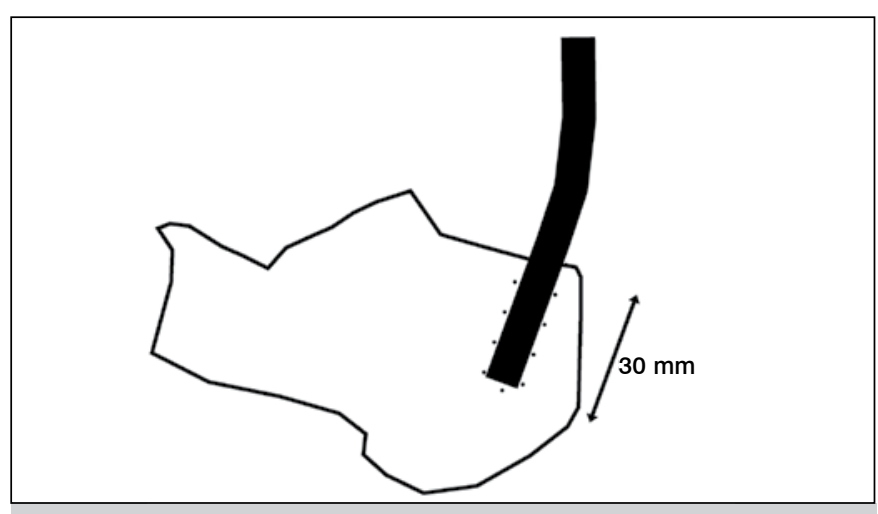

Figure 3. Thirty millimeters tunnel for $\mathrm{FHL}$ reinsertion using interference screws.

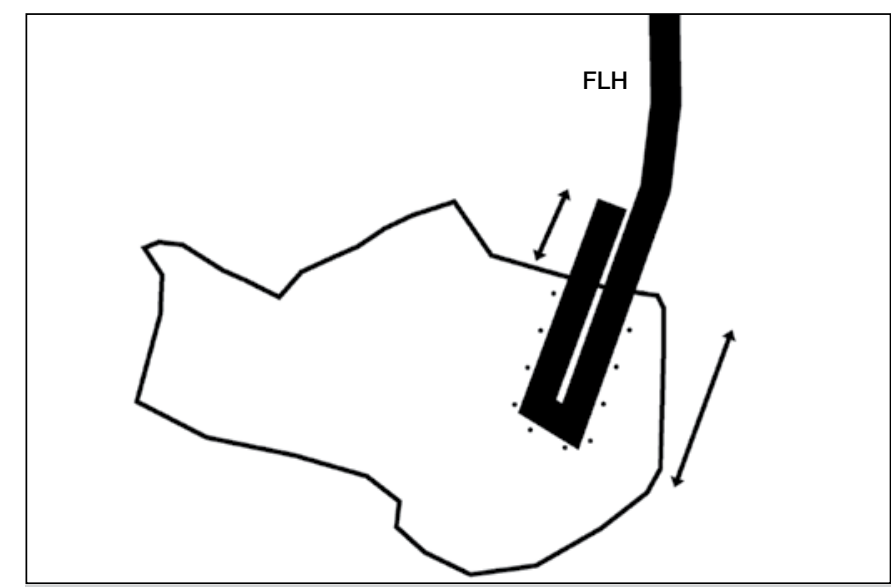

Figure 4. Simulation of reintegration with $\mathrm{FHL}$ interference screw removed by double track; in this condition there is a tendon length gain of $42 \mathrm{~mm}$ gain, of which $30 \mathrm{~mm}$ are within the tunnel and only $12 \mathrm{~mm}$ are available for use.

making, thus, a bridge that fills the area of the resected pathological tendon. The bridge, thus, contains a double FHL graft, a band in downward direction, and another in the upward tendon. (Figure 5) The reconstruction performed in such a way is suficiente ${ }^{16}$ and the needed tendon length depends on the size of the distal stump, and the remaining 


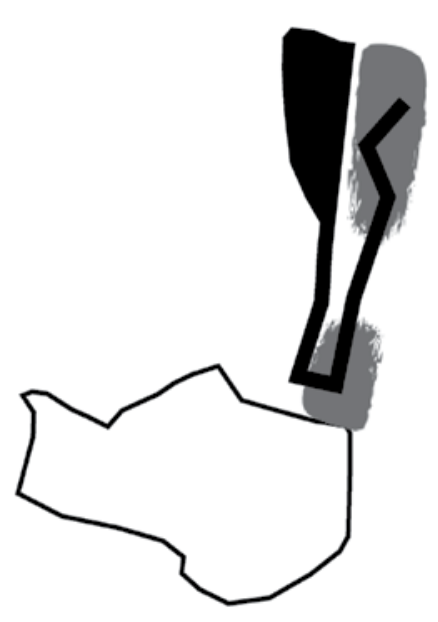

Figure 5. Reconstruction of the Achilles tendon injured by non insertional pathology. The tendon defect is filled by a double graft. defect after debridement of the Achilles tendon. Having a $42 \mathrm{~mm}$ GTL may be necessary or not, depending on the length of this defect.

The weakness of the study lies in the fact that no bone tunnels were made in the calcaneus to assess the influence of the FHL length in the simulation with different types of its reintegration. The values used in the discussion are reported and there are not the ones found in our bodies. The importance of this work lies in the fact that it is the first paper to quantify the difference in length of the FHL graft obtained by single or double track in the Brazilian population.

\section{CONCLUSION}

Dissection of $\mathrm{FHL}$ for tendon transfer, by double access provides a $42 \mathrm{~mm}$ graft length gain, in comparison to the single track procedure. Thus, depending on the particular case chosen for each technique, the surgeon must choose using one or two access tracks, as he could rely on extra $4 \mathrm{~cm}$ of the transferred tendon.

\section{REFERENCES}

1. DeMaio M, Paine R, Drez DJ Jr. Achilles tendonitis. Orthopedics. 1995;18(2):195-204.

2. Wapner KL, Pavlock GS, Hecht PJ, Naselli F, Walther R. Repair of chronic Achilles tendon rupture with flexor hallucis longus tendon transfer. Foot Ankle. 1993;14(8):443-9.

3. Silver RL, de la Garza J, Rang M. The myth of muscle balance. A study of relative strengths and excursions of normal muscles about the foot and ankle. J Bone Joint Surg Br. 1985;67(3):432-7.

4. Richardson DR, Willers J, Cohen BE, Davis WH, Jones CP, Anderson RB. Evaluation of the hallux morbidity of single-incision flexor hallucis longus tendon transfer. Foot Ankle Int. 2009;30(7):627-30.

5. Mulier T, Rummens E, Dereymaeker G. Risk of neurovascular injuries in flexor hallucis longus tendon transfers: an anatomic cadaver study. Foot Ankle Int. 2007;28(8):910-5.

6. Hansen ST. Trauma to the heel cord. In: Jahss MH, editor. Disorders of the foot and ankle. 2nd ed. Philadelphia: Saunders; 1991. p. 2357.

7. Den Hartog BD. Flexor hallucis longus transfer for chronic Achilles tendonosis. Foot Ankle Int. 2003;24(3):233-7.

8. Elias I, Besser M, Nazarian LN, Raikin SM. Reconstruction for missed or neglected Achilles tendon rupture with $\mathrm{V}$-Y lengthening and flexor hallucis longus tendon transfer through one incision. Foot Ankle Int. 2007;28(12):1238-48.

9. Elias I, Raikin SM, Besser MP, Nazarian LN. Outcomes of chronic insertional Achilles tendinosis using FHL autograft through single incision. Foot Ankle Int. 2009;30(3):197-204.

10. Hahn F, Meyer P, Maiwald C, Zanetti M, Vienne P. Treatment of chronic Achilles tendinopathy and ruptures with flexor hallucis tendon transfer: clinical outcome and
MRI findings. Foot Ankle Int. 2008;29(8):794-802

11. Mahajan $\mathrm{RH}$, Dalal RB. Flexor hallucis longus tendon transfer for reconstruction of chronically ruptured Achilles tendons. J Orthop Surg (Hong Kong). 2009;17(2):194-8.

12. Martin RL, Manning CM, Carcia CR, Conti SF. An outcome study of chronic Achilles tendinosis after excision of the Achilles tendon and flexor hallucis longus tendon transfer. Foot Ankle Int. 2005;26(9):691-7.

13. Wegrzyn J, Luciani JF, Philippot R, Brunet-Guedj E, Moyen B, Besse JL. Chronic Achilles tendon rupture reconstruction using a modified flexor hallucis longus transfer. Int Orthop. 2010;34(8):1187-92.

14. Wilcox DK, Bohay DR, Anderson JG. Treatment of chronic achilles tendon disorders with flexor hallucis longus tendon transfer/augmentation. Foot Ankle Int. 2000;21(12):1004-10.

15. Tashjian RZ, Hur J, Sullivan RJ, Campbell JT, DiGiovanni CW. Flexor hallucis longus transfer for repair of chronic achilles tendinopathy. Foot Ankle Int. 2003;24(9):673-6.

16. Hahn F, Maiwald C, Horstmann T, Vienne P. Changes in plantar pressure distribution after Achilles tendon augmentation with flexor hallucis longus transfer. Clin Biomech (Bristol, Avon). 2008;23(1):109-16.

17. Coull R, Flavin R, Stephens MM. Flexor hallucis longus tendon transfer: evaluation of postoperative morbidity. Foot Ankle Int. 2003;24(12):931-4.

18. DeCarbo WT, Hyer CF. Interference screw fixation for flexor hallucis longus tendon transfer for chronic Achilles tendonopathy. J Foot Ankle Surg. 2008;47(1):69-72.

19. Sarrafian SK, Kelikian AS. Osteology. In: Kelikian AS, editor. Sarrafian's anatomy of the foot and ankle. Philadelphia: Lippincott Williams \& Wilkins; 2011. p. 40-8. 